brain stimulation cannot be adequately examined at present, we are forced to analyze ESB within the traditional categories of motivational variables. It is traditionally assumed that the drive variable relates to changes in organismic states produced by deprivation conditions and/or strong stimulation, while the incentive variable relates to the nature of the reward and to its delivery. Although Gallistel's definition of drive (i.e., "... whatever produces changes in the direction and avidity of behavior in the absence of changes in the animal's knowledge of the reward contingencies") does in general account for this differentiation, it fails to specify the variables which contribute to drive, and also includes response directionality as a consequence of drive manipulations rather than as a consequence of incentive related stimuli as has traditionally been done.

It is our contention that the proper differentiation of drive and incentive variables is crucial for understanding the nature of performance for ESB. More importantly, the distinction between the two positions, induced "drive" vs reward-induced incentive, can be better appreciated by the different predictions which they generate. Possibly the most difficult data for the drive-decay position to assimilate was presented by Panksepp \& Trowill (1968) in their demonstration that, under special training conditions, animals show the highest extinction responding after a period of no reward rather than immediately after a period of reinforcement. A forthcoming review summarizes other data difficult for the drive decay position to explain (Trowill, Panksepp, \& Gandelman, in press).

The drive-decay position has led to interesting predictions (e.g., extinction without responding) which were not previously noted in the behavior of animals working for food or water. The incentive position does not deny such phenomena; rather, it suggests that they are transient phenomena associated with responding for strong rewards, of any type, under the conditions of nondeprivation (Panksepp \& Trowill, 1967).

Gallistel also cites the provocative results of Deutsch which purport to demonstrate parallel, but separate, fiber pathways in the brain which control "reward" and "drive." While we do not question Deutsch's results, we do question his interpretation. There may be many functional purposes served by parallel-running pathways with differing fiber refractory times. For example, such parallel paths could represent centripetal (sensory-afferents) and centrifugal (sensory-efferents) fiber bundles. The relevance of Deutsch's elegant experiments thus seems unclear.

Gallistel also takes issue with our citation of Scott's data (1967). As far as we can see, Scott's data only serve to weaken the drive-decay hypothesis. The crucial factor in discussing Scott's data in relation to our own is not in the fact that our animals still showed overnight decrements, which is common with conventional rewards, especially under low-deprivation conditions, but that with Scott's procedure (i.e., much practice with long ITIs), the decremental effect of even 24-h ITIs could be overcome. In fact, Scott felt that the running speed of his animals after 24-h intervals was comparable to those reported for massed trials. During the last postshift days our animals also showed no differences in running speed despite the differences in ITIs. A rigorous drive-decay explanation should, we believe, predict that after a $24-\mathrm{h}$ interval Ss should show little motivation to perform at all, no matter what the training conditions. At the very least, the initial trials of each day should always show an enormous performance decrement and should never attain the speeds emitted during massed practice.

\section{REFERENCES}

DEUTSCH, J. A., \& HOWARTH, C. I. Some tests of a theory of intracranial self-stimulation. Psychological Review, 1963, 70, 444-460.
GALLISTEL, C. R. Electrical self-stimulation and its theoretical implications. Psychological Bulletin, 1964, 61, 23-34.

GALLISTEL, C. R. Intracranial stimulation and natural reward: Differential effects of trial spacing. Psychonomic Science, 1967, 9, 167-168.

GALLISTEL, C. R. Comments on Panksepp et al. Psychonomic Science, 1969, 00, 000-000.

PANKSEPP, J., GANDELMAN, R., \& TROWILL, $J$. The effect of intertrial interval on running performance for ESB. Psychonomic Science, $1968,13,135-136$.

PANKSEPP, J., \& TROWILL, J. A. Intraoral self-injection: II. The simulation of selfstimulation phenomenon with a conventional reward. Psychonomic Science, 1967, 9, 407-408.

PANKSEPP, J., \& TROWILL, J. A. Extinction following intracranial reward: Frustration or drive decay? Psychonomic Science, 1968, 12, 173-174.

PANKSEPP, J., \& TROWILL, J. A. Positive and negative contrast effects with hypothalamic reward. Physiology and Behavior, 1969, 4, 173-175.

SCOTT, J. W. Brain stimulation reinforcement with distributed practice: Effects of electrode locus, previous experience and stimulus intensity. Journal of Comparative \& Physiological Psychology, 1967,63, 175-183.

TROWILL, J. A., PANKSEPP, J., \& GANDELMAN, $R$. An incentive model of rewarding brain stimulation. Psychological Review, in press.

\title{
Drug effects on mouse exploratory behavior'
}

\section{HAROLD G. WAKELEY and DENNIS O'SULLIVAN, IIT Research Institute, Chicago, Ill. 60616}

Ditran, atropine sulfate, d-amphetamine, chlorpromazine, or isotonic saline were administered intraperitoneally to $B D F_{1}$ mice prior to measurement of exploratory activity. D-amphetamine and chlorpromazine decreased exploration, whereas the anticholinergic compounds Ditran and atropine sulfate increased exploratory activity.

Primary screening tests that differentiate the effects of anticholinergic drugs from the effects of general stimulant or depressant drugs on animals are of value in assaying compounds for potential psychotropic effects in humans. Screening methods used for anticholinergic compounds have included measures of anorexia (Cohen, 1965) or changes in spontaneous activity (Lipman et al, 1963). Conventional locomotor or spontaneous activity tests ascribe increased activity to both anticholinergic compounds and stimulants such as amphetamine (Tripod et al, 1954). Observations of anticholinergic drug-induced changes in responsiveness to stimuli indicated that measurement of exploratory activity rather than locomotor activity might differentiate anticholinergic compounds from stimulants (Abood et al, 1959).

Carlton (1963) has suggested that a cholinergic system in the brain antagonizes a catecholamine system than activates behavior. The cholinergic antagonism is selective and proportional to the degree that behavior is unrewarded. According to this hypothesis, anticholinergic compounds should increase responsiveness to stimuli. Compounds that affect the catecholamine system, such as d-amphetamine, should shift the activity level, though not necessarily the response to specific stimuli.

An appropriate test should differentiate between the change in responsiveness to stimuli induced by anticholinergics and the change in activation level induced by compounds affecting the catecholamine system. The exploratory behavior test of Boissier \& Simon (1964) was selected. Exploration is defined as the performance of a quantifiable investigatory behavior pattern in response to specific stimuli, as contrasted with generalized locomotor activity.

To determine whether anticholinergic 


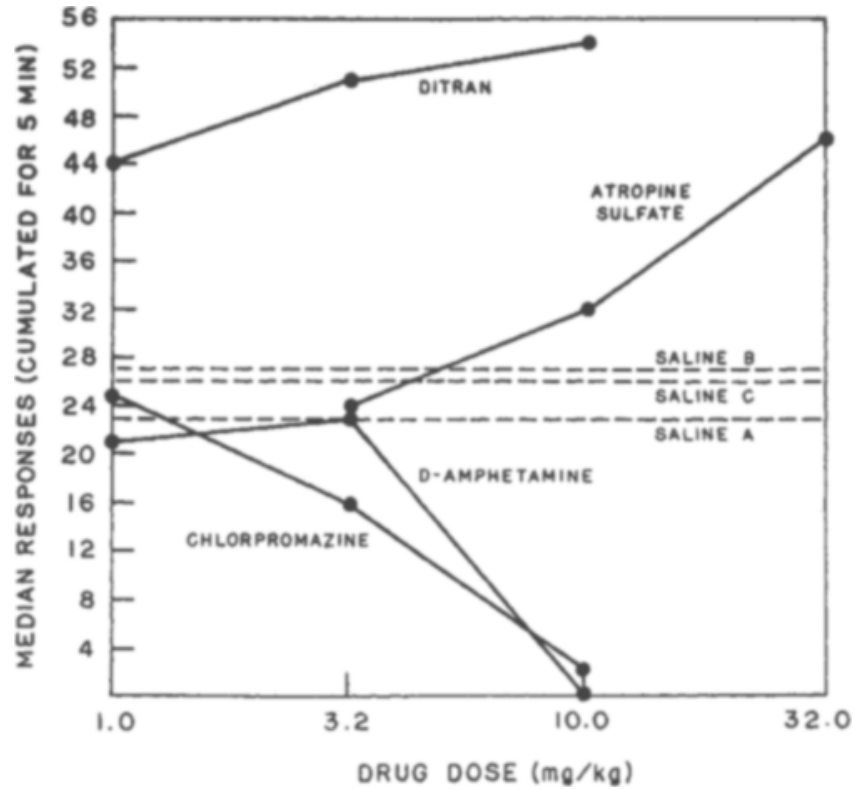

drugs have a quantitative effect on exploratory behavior, two anticholinergic drugs with different potencies, Ditran and atropine sulfate, were selected. Chlorpromazine and d-amphetamine were selected as compounds that inhibit and facilitate cholinolytic activity, respectively (Irwin, 1961).

\section{SUBJECTS}

The Ss were male $\mathrm{BDF}_{1}$ mice, 25- to $30-\mathrm{g}$ body weight. The mice were housed 10 per cage in a $74 \operatorname{deg} F$ room with a 12-h light/dark cycle for at least 7 days prior to testing. Food and water were provided ad lib.

\section{APPARATUS}

The apparatus was based on the design of Boissier \& Simon (1964). It consists of a gray $1 / 2$-in. plywood board, 16 in. square,


board was supported 12 in. above a slate table top by $4 \frac{1}{2}$-in. diam aluminum legs. $A$ circular fluorescent lamp (Swingo-lite Model BBM-9) centered 12 in. above the board was the only illumination. Testing was performed in a sound-dampened room, and the 0 was seated $6 \mathrm{ft}$ from the test board.

\section{PROCEDURE}

Three saline control groups and three dose levels, in 0.5-log increments, of Ditran, atropine sulfate, d-amphetamine, and chlorpromazine were used. Fifteen mice were tested each day, one from each group and 19 replicates per group. The order of testing mice from each group was randomized. To reduce the possibility of $\mathrm{E}$ bias (Rosenthal, 1964), blind procedure was used for all injections. The drugs and saline were administered intraperitoneally with a 15-min latency period between injection and testing. The mice were randomly assigned to an experimental or control group on the test day; each mouse was used once. The drug solutions were prepared weekly and stored at $-10 \operatorname{deg} \mathrm{C}$ to minimize chemical decomposition. A preliminary experiment with mice injected with saline demonstrated that there is no significant day-to-day variation in exploratory behavior with these procedures.

Testing was performed by placing a mouse in the center of the board and recording the number of times during a 5-min period that the mouse inserted its head into a hole such that his eyes were below the upper surface of the board (poke). Repeated pokes in the same hole were recorded only when the head was completely withdrawn between pokes. Aberrations in general behavior or disposition were recorded descriptively for later comparison with the drug dose administered. Results were analyzed by the Mann-Whitney test.

\section{RESULTS AND DISCUSSION}

The median for 19 observations at each dose is plotted in Fig. 1. There was no significant difference among the saline control groups (A, B, and C) at the 0.80 level, indicating that stable control values were obtained. Mice injected with the drugs showed significantly different exploratory activity at the 0.01 level or less when given the following doses: Ditran at all doses, atropine sulfate at $32.0 \mathrm{mg} / \mathrm{kg}$, chlorpromazine at $10.0 \mathrm{mg} / \mathrm{kg}$, and d-amphetamine at $10.0 \mathrm{mg} / \mathrm{kg}$. Typical dose response curves for all drugs were obtained. The mice administered d-amphetamine at $10 \mathrm{mg} / \mathrm{kg}$ uniformly exhibited continuous movement around the periphery of the board, in addition to piloerection and high carriage. The chlorpromazine-induced response decrement was accompanied by a decrease in locomotor activity and low carriage. A response to stimuli persisted since attempts to poke were still evident. The two anticholinergic compounds, Ditran and atropine sulfate, increased exploratory
Fig. 1. Effect of drugs on exploratory (poke) behavior in $\mathrm{BDF}_{1}$ mice.

activity. The difference in potency between the two drugs may be due to the relative ease of passage through the blood-brain barrier, differences in central activity, or their effect on a number of other factors described by Abood et al (1959).

Carlton's hypothesis that a cholinergic system mediates responsiveness to stimuli and that a catecholamine system mediates activation level is supported by this experiment. The two compounds, d-amphetamine and chlorpromazine, that affect the catecholamine system decreased exploratory behavior, whereas locomotor activity was differentially affected by the two drugs. D-amphetamine, which mimics the action of catecholamines, increased locomotor activity, whereas chlorpromazine decreased spontaneous activity. These results indicate that catecholamines may control activation level. The anticholinergic drugs increased exploratory behavior, indicating that a cholinergic system may mediate responsiveness to stimuli.

Aside from the theoretical applications of this experiment, it has been demonstrated that a test of exploratory behavior can discriminate between anticholinergic drugs of different potency.

\section{REFERENCES}

ABOOD, L. G., OSTFELD, A., \& BIEL, J. H. Structure-activity relationships of 3-piperidyl benzilates with psychotogenic properties. Archives Internationales de Pharmacodynamie, $1959,120,186-200$.

BOISSIER, J. R., \& SIMON, P. Dissociation de deux composantes le comportment d'investigation de la souris. Archives Internationales de Pharmocodynamie, 1964, 47, 372-387.

CARLTON, P. L. Cholinergic mechanisms in the control of behavior by the brain. Psychological Review, 1963, 70, 19-39.

COHEN, M. A feeding method for evaluating anticholinergic psychomimetic drugs. Archives Internationales de Pharmacodynamie, 1966, $161,120-125$.

IRWIN, S. The actions of drugs on psychomotor activity. Review of Canadian Biology, 1961, 20, 239-250.

LIPMAN, V. C., SHURRAGER, P. S., \& ABOOD, L. G. Effect of anticholinergic psychotomimetics on motor activity and body temperature. Archives Internationales de Pharmacodynamie, 1963, 146, 174-191.

ROSENTHAL, R. Experimenter outcomeorientation and the results of the psychological experiment. Psychological Bulletin, 1964, 61, 405-412.

TRIPOD, J., BEIN, J., \& MEIER, R. Characterization of central effects of Serpasil (reserpine, a new alkaloid of Rawolfia serpentine $B$.) and their antagonistic reactions. Archives Internationales Pharmacodynamie, 1954, 96, 406-425.

\section{NOTE}

1. Supported by IIT Research Institute, Chicago, Illinois. Ditran was generously supplied by Lakeside Laboratories, Milwaukee, Wis. The advice of Dr. M. Cohen and Dr. R. Sroges is gratefully acknowledged. 